\title{
Testing Signal Enhancement Mechanisms in the Dissolution NMR of Acetone
}

\author{
Javier Alonso-Valdesueiro ${ }^{\mathrm{a}}$, Stuart J. Elliott ${ }^{\mathrm{a}}$, Christian Bengs ${ }^{\mathrm{a}}$, Benno Meier ${ }^{\mathrm{a}}$, Malcolm H. Levitt ${ }^{\mathrm{a}, *}$ \\ ${ }^{a}$ School of Chemistry, University of Southampton, Southampton SO17 1BJ, United Kingdom
}

\begin{abstract}
In cryogenic dissolution NMR experiments, a substance of interest is allowed to rest in a strong magnetic field at cryogenic temperature, before dissolving the substance in a warm solvent, transferring it to a high-resolution NMR spectrometer, and observing the solution-state NMR spectrum. In some cases, negative enhancements of the ${ }^{13} \mathrm{C}$ NMR signals are observed, which have been attributed to quantum-rotor-induced polarization. We show that in the case of acetone (propan-2-one) the negative signal enhancements of the methyl ${ }^{13} \mathrm{C}$ sites may be understood by invoking conventional cross-relaxation within the methyl groups. The ${ }^{1} \mathrm{H}$ nuclei acquire a relative large net polarization through thermal equilibration in a magnetic field at low temperature, facilitated by the methyl rotation which acts as a relaxation sink; after dissolution, the ${ }^{1} \mathrm{H}$ magnetization slowly returns to thermal equilibrium at high temperature, in part by crossrelaxation processes, which induce a transient negative polarization of nearby ${ }^{13} \mathrm{C}$ nuclei. We provide evidence for this mechanism experimentally and theoretically by saturating the ${ }^{1} \mathrm{H}$ magnetization using a radiofrequency field pulse sequence before dissolution and comparing the ${ }^{13} \mathrm{C}$ magnetization evolution after dissolution with the results obtained from a conventional ${ }^{1} \mathrm{H}_{-}{ }^{13} \mathrm{C}$ cross relaxation model of the $\mathrm{CH}_{3}$ moieties in acetone.
\end{abstract}

Keywords: methyl groups, cross-relaxation, quantum rotor polarization, dissolution, nuclear Overhauser effect, NOE

\section{Introduction}

In cryogenic dissolution-NMR experiments, a sample is allowed to equilibrate at cryogenic temperatures, often in a strong magnetic field. The cold sample is dissolved rapidly in a warm solvent, and the NMR signals observed. In some cases, this leads to strongly enhanced solution-state NMR signals for ${ }^{13} \mathrm{C}$ nuclei. This phenomenon has been attributed to spin polarization effects associated with the quantized rotation of low-barrier methyl groups in the solid state, i.e. quantum-rotor induced polarization (QRIP) [17]. The NMR enhancements are relatively modest (a factor of $\sim 100$ or less), but are observed in the absence of radical doping and microwave irradiation, as opposed to the related dissolution-DNP (dynamic nuclear polarization) experiment which may give rise to much larger enhancements [8].

There are two classes of radical-free dissolution-NMR observations which have been attributed to QRIP, and which are termed here the "Birmingham" and "Leipzig" effects.

The Birmingham-based group of Ludwig et al. reported negative enhancements of ${ }^{13} \mathrm{C}$ NMR signals in dissolutionNMR experiments, for a wide range of materials containing methyl groups [1]. The observed enhancements are relatively small ( $\sim 18$ was reported for acetone) and typically require long equilibration of the sample in the polarizer (tens of hours at $1.4 \mathrm{~K}$ ) in a high magnetic field $(3 \mathrm{~T})$

\footnotetext{
*Corresponding author

Email address: mhl@soton.ac.uk (Malcolm H. Levitt)
}

prior to dissolution. The negative enhancements of the solution-state ${ }^{13} \mathrm{C}$ spectrum are reported in the presence of ${ }^{1} \mathrm{H}$ decoupling, implying an in-phase ${ }^{1} \mathrm{H}$-coupled ${ }^{13} \mathrm{C}$ spectral multiplet (as verified below). The effects were attributed to QRIP, even though most of the compounds used in the Birmingham study are not known to exhibit the low-barrier methyl rotation in the cryogenic state which is considered to be a prerequisite for quantum-rotor phenomena $[9-11]$.

The Leipzig group of Icker et al. [2-4] also observed strong enhancements of the ${ }^{13} \mathrm{C}$ and ${ }^{1} \mathrm{H} \mathrm{NMR}$ in dissolution-NMR experiments. However, unlike the "Birmingham" phenomena, the "Leipzig" effects were observed for samples cooled in the ambient magnetic field, outside a strong magnet. Relatively strong (>100) antiphase signal enhancements were obtained for the ${ }^{1} \mathrm{H}$ coupled ${ }^{13} \mathrm{C}$ multiplet, which could only be observed in the absence of ${ }^{1} \mathrm{H}$ decoupling. The "Leipzig" effects were particularly prominent for compounds known to have large methyl tunnelling splittings in the cryogenic solid state, such as 4-methylpyridine (also known as $\gamma$-picoline).

A detailed QRIP mechanism for the "Leipzig" effects was proposed $[5,6]$, involving the generation of a strong population imbalance between the $A$ and $E$ symmetry species of the methyl quantum rotor at low temperature. The A/E population imbalance substantially persists during the dissolution process and leads to a hyperpolarized long-lived state (LLS) for the methyl groups at high temperature. The LLS slowly returns to equilibrium in the ambient temperature solution. In the case of ${ }^{13} \mathrm{C}$-bearing 
methyl groups, the return to equilibrium is driven in part by ${ }^{1} \mathrm{H}-{ }^{13} \mathrm{C}$ cross-relaxation. The generation of an enhanced antiphase ${ }^{13} \mathrm{C}$ multiplet has been modelled in detail, including cross-correlation effects [6]. The QRIP mechanism of the Leipzig effects was probed by further experiments involving the application of selective radiofrequency pulses [7].

In contrast to the "Leipzig" effects, the mechanism of the earlier "Birmingham" phenomena is not wellestablished. The quantum-rotor mechanism initially proposed by Ludwig et al. [1] is suspect, since many of the materials employed in the "Birmingham" experiments are known through numerous physical measurements to have methyl groups which are rotationally hindered and which therefore have vanishingly small tunneling splittings $[12,13]$ - often much smaller than the nuclear Zeeman splitting under identical conditions. It is hard to see how a significant QRIP could arise for such materials, without the use of temperatures in the $\mathrm{mK}$ range.

Dumez et al. [6] proposed an alternative mechanism for the observed negative signal enhancements in the "Birmingham" experiments. The mechanism involves the following elements: (1) The role of the $\mathrm{CH}_{3}$ groups is not to provide quantum-rotor polarization, but to act as passive spin-lattice relaxation sinks allowing ${ }^{1} \mathrm{H}$ polarization to build up along the magnetic field under cryogenic conditions, in a reasonable time; (2) Since the degree of thermal spin polarization is inversely proportional to the absolute temperature, the transfer of a sample that has reached thermal equilibrium at low temperature to a hightemperature environment leads to a proton spin system that is far from thermal equilibrium at the higher temperature; (3) The return of the proton system to thermal equilibrium in solution involves, in part, dipole-dipole cross-relaxation with the nearby ${ }^{13} \mathrm{C}$ nuclei. For the small molecules involved in the "Birmingham" experiments, the cross-relaxation is dominated by double-quantum processes, leading to a negative magnetization enhancement of the nearby ${ }^{13} \mathrm{C}$ sites, through a conventional heteronuclear nuclear Overhauser effect (NOE) [14].

In this paper, we provide evidence of the cross-relaxation mechanism for the "Birmingham" effects [1]. The results presented in ref. [1] for acetone are revisited and extended with experiments in which the trajectory of the ${ }^{13} \mathrm{C}$ magnetization is followed at high field after dissolution. We show that the sign of the enhanced ${ }^{13} \mathrm{C}$ signals is changed when the ${ }^{1} \mathrm{H}$ magnetization is destroyed in the cryogenic solid state prior to dissolution. This behavior is successfully modeled by dipole-dipole cross-relaxation between the $\mathrm{CH}_{3}$ proton and carbon nuclei in the extreme narrowing limit. The observed trajectories of ${ }^{13} \mathrm{C}$ magnetization are successfully reproduced, both with and without ${ }^{1} \mathrm{H}$ saturation prior to dissolution. Therefore, at least in the case of acetone, there is no need to invoke an unconventional QRIP mechanism.

\section{Methods}

\subsection{Sample}

Samples of acetone (propan-2-one) were used. In most cases the acetone was unlabelled, with the ${ }^{13} \mathrm{C}$ nuclei observed in natural abundance. In some experiments, a 1:1 mixture of unlabelled acetone and $\left[1,3-{ }^{13} \mathrm{C}_{2}\right]$-acetone was used in order to enhance the ${ }^{13} \mathrm{C}$ signal-to-noise ratio.

The ${ }^{13} \mathrm{C}$ spectrum of acetone at $298 \mathrm{~K}$ presents two ${ }^{13} \mathrm{C}$ resonances at $206.7 \mathrm{ppm}\left(\mathrm{CO}\right.$ site) and $29.9 \mathrm{ppm}\left(\mathrm{CH}_{3}\right.$ site). Both shifts were referenced to the methanol $\mathrm{CH}_{3}$ peak, assumed to be at $49.2 \mathrm{ppm}$ relative to tetramethylsilane.

\subsection{Apparatus}

The experiments employed a home-built dissolutionDNP polarizer based on the design by Ardenkjaer-Larsen et al. [8], but not employing the microwave elements.

The dissolution apparatus employs a commercial flowthrough cryostat mounted in the room-temperature bore of a $6.7 \mathrm{~T}$ superconducting magnet. A base temperature of $\sim 1.6 \mathrm{~K}$ may be achieved by flooding the cryostat with liquid He and reducing the pressure using a pump. However, all experiments described here were performed using liquid He without pumping, providing a sample temperature of $4.2 \mathrm{~K}$.

The cryogenic chamber at the base of the cryostat accommodates a PTFE sample cup with $120 \mu \mathrm{L}$ capacity. A saddle coil tuned to the ${ }^{1} \mathrm{H}$ resonance frequency of $285 \mathrm{MHz}$ surrounds the sample. The ${ }^{1} \mathrm{H}$ coil provides a $90^{\circ}$ pulse length of $17.5 \mu$ s when using $15 \mathrm{~W}$ of radiofrequency power, at a sample temperature of $4.2 \mathrm{~K}$.

The dissolution stick is equipped with a pressure cooker for the dissolution solvent. After insertion into the cryostat, the pressurised hot solvent is flushed through the cryogenic sample cup, chased by 7 bar helium gas. The solution exits the cryostat through a 1/8 inch PTFE tube and flows through a $2.25 \mathrm{~m}$ tunnel lined with permanent magnets [15], generating a field of $\sim 0.74 \mathrm{~T}$ perpendicular to the flow direction. The sample emerges from the tunnel and is loaded into a receiver device located $20 \mathrm{~cm}$ above the sweet spot of a high-resolution 11.7 NMR magnet, as previous designs $[16,17]$. The sample is injected into the NMR tube, after allowing a few seconds for dissipation of the He gas.

Although the field inside the magnetic tunnel is $\sim 0.74 \mathrm{~T}$, the field at the entrances of the two magnets is considerably lower $(\sim 3 \mathrm{mT})$, since both of our NMR magnets are shielded. The magnetic field and temperature trajectories of the sample during the dissolution and transport processes are sketched qualitatively in Figure 1.

\subsection{Procedure}

All experiments were performed with $100 \mu \mathrm{L}$ samples of acetone which were allowed to rest for $\sim 90 \mathrm{~min}$ at $4.2 \mathrm{~K}$ in the $6.7 \mathrm{~T}$ polarizer field before the start of each experiment. 


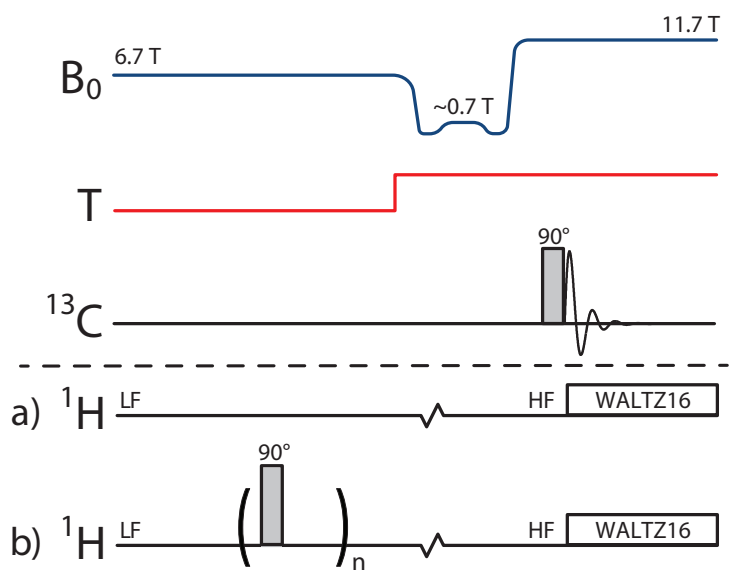

Figure 1: Experimental procedure for investigating the "Birmingham" polarization effects. Top: qualitative trajectories of the magnetic field and temperature. The temperature jump indicates dissolution. The time axis is not to scale: The waiting time in the cryostat is $\sim 90 \mathrm{~min}$ while the dissolution and sample transport takes $\sim 8-15$ seconds. A $90^{\circ}{ }^{13} \mathrm{C}$ pulse is applied after stabilisation of the solution in high magnetic field and the free-induction decay detected in the presence of WALTZ-16 ${ }^{1} \mathrm{H}$ decoupling [18]. (a) No manipulations are performed before dissolution; (b) $\mathrm{A}^{1} \mathrm{H}$ saturation sequence of $51290^{\circ}$ pulses, each separated by $100 \mathrm{~ms}$, is applied to the cryogenic sample before dissolution.

In some cases, radiofrequency pulses were applied to the cold sample using the $285 \mathrm{MHz}{ }^{1} \mathrm{H}$ coil in the $6.7 \mathrm{~T}$ magnet, immediately prior to dissolution.

Dissolution was achieved by heating $4 \mathrm{~mL}$ of methanol $\left(\mathrm{CH}_{3} \mathrm{OH}\right)$ to $\sim 180^{\circ} \mathrm{C}$ in the pressure cooker at 7 bar. The sample was lifted above the liquid-He reservoir, the dissolution stick inserted, and the hot solvent injected. The warm methanol/acetone mixture was driven out of the cryostat, through the magnetic tunnel, and into the receiver mounted in the high-resolution $11.7 \mathrm{~T}$ magnet. The sample was injected into the NMR tube, after allowing a few seconds for the dissipation of He gas. The transfer time was approximately 8 to 15 seconds from dissolution to NMR signal acquisition.

In all cases, a thermal equilibrium spectrum was acquired in the $11.7 \mathrm{~T}$ magnet about 10 minutes after the conclusion of the experiment, by accumulating 8 transients generated by ${ }^{13} \mathrm{C} 90^{\circ}$ pulses, with a waiting time of $120 \mathrm{~s}$ between transients. The ${ }^{13} \mathrm{C}$ signal enhancements were estimated by comparing the spectral integrals with those of the thermal equilibrium spectrum, taking into account the number of transients and the pulse flip angles.

\section{Experiments and Results}

\subsection{Spin-lattice relaxation at $4.2 \mathrm{~K}$}

The ${ }^{1} \mathrm{H}$ spin-lattice relaxation of acetone was observed at $4.2 \mathrm{~K}$ and $6.7 \mathrm{~T}$ by saturation-recovery. The relaxation was found to be non-exponential, with a dominant $T_{1}$ value of $\sim 20 \mathrm{~min}$. The non-exponential relaxation behaviour might reflect the existence of domains with different relaxation times. The ${ }^{13} \mathrm{C}$ spin-lattice relaxation time constant could not be estimated accurately but was significantly slower than the ${ }^{1} \mathrm{H}$ relaxation.
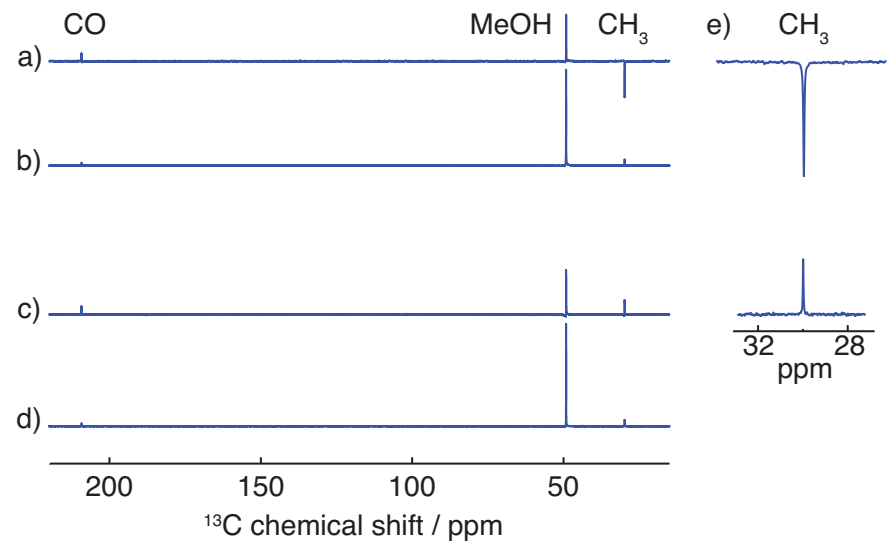

Figure 2: ${ }^{1} \mathrm{H}$-decoupled ${ }^{13} \mathrm{C}$ spectra acquired for the experiments in Figure 1. The ${ }^{13} \mathrm{C}$ peaks of the acetone $\mathrm{CO}$ and $\mathrm{CH}_{3}$ sites, and the ${ }^{13} \mathrm{C}$ peak of the methanol solvent, are indicated. (a) Spectrum acquired by performing the experiment in Fig 1(a). (b) Thermal-equilibrium spectrum acquired 10 minutes after (a) by accumulating 8 transients induced by a ${ }^{13} \mathrm{C} 90^{\circ}$ pulse, separated by $120 \mathrm{~s}$. (c) Spectrum acquired by performing the experiment in Figure 1(b). (d) Thermal-equilibrium spectrum acquired 10 minutes after (c) by accumulating 8 transients induced by a ${ }^{13} \mathrm{C} 90^{\circ}$ pulse, separated by $120 \mathrm{~s}$. (e) Expanded views of the acetone $\mathrm{CH}_{3}$ peaks from (a) and (c).

\subsection{Dissolution-Induced Signal Inversion}

The procedure sketched in Figure 1(a) was used in an attempt to reproduce the "Birmingham" effects. Unlabelled acetone was used. No ${ }^{1} \mathrm{H}$ pulses were applied to the sample prior to dissolution. After dissolution, transport and injection, the ${ }^{13} \mathrm{C}$ signal was induced by a single ${ }^{13} \mathrm{C}$ $90^{\circ}$ pulse and acquired in the presence of WALTZ-16 ${ }^{1} \mathrm{H}$ decoupling [18]. The resulting spectrum is shown in Figure 2(a) and displays an enhanced and inverted ${ }^{13} \mathrm{C}$ peak for the $\mathrm{CH}_{3}$ site. The ${ }^{13} \mathrm{C}$ peaks of the $\mathrm{CO}$ acetone site and the methanol solvent are not inverted. A thermal equilibrium spectrum, obtained immediately after the dissolution experiment, is shown in Figure 2(b). A comparison shows that the ${ }^{13} \mathrm{C}$ signal of the $\mathrm{CH}_{3}$ site in (a) is inverted in sign and enhanced by a factor of $\sim 5$ relative to the thermal equilibrium spectrum.

Although these findings are in qualitative agreement with the Birmingham observations [1], the negative enhancement of the $\mathrm{CH}_{3}$ signal for acetone is a factor of $\sim 3$ smaller than that reported by Ludwig et al. [1]. Possible reasons for this discrepancy are discussed below.

We performed the same experiment but without ${ }^{1} \mathrm{H}$ decoupling during ${ }^{13} \mathrm{C}$ signal acquisition, in order to explore the form of the ${ }^{1} \mathrm{H}$-coupled ${ }^{13} \mathrm{C}$ multiplet. This experiment was performed on a 1:1 mixture of unlabelled acetone and $\left[1,2-{ }^{13} \mathrm{C}_{2}\right]$-acetone, in order to enhance the signal-to-noise ratio. The resulting spectrum is shown in Figure 3(a) and shows an enhanced and inverted ${ }^{13} \mathrm{C}$ quartet, with the normal structure and intensity ratios expected for a $\mathrm{CH}_{3}$ group, with additional minor splittings associated with the ${ }^{13} \mathrm{C}-{ }^{13} \mathrm{C}$ J-coupling. There is no evidence of the intensity alternations and distortions expected for a quantum-rotor mechanism [2-7]. 


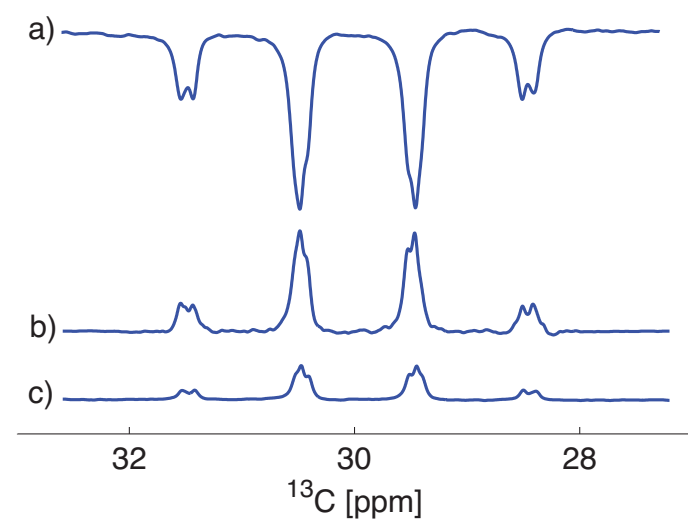

Figure 3: $\quad \mathrm{CH}_{3}$ region of ${ }^{13} \mathrm{C}$ spectra for a 1:1 mixture of unlabelled acetone and $\left[1,2-{ }^{13} \mathrm{C}_{2}\right]$-acetone. (a) Procedure in Figure 1 (a) but without ${ }^{1} \mathrm{H}$ decoupling during acquisition. (b) Procedure in Figure 1(b) but without ${ }^{1} \mathrm{H}$ decoupling during acquisition. (c) Spectrum obtained on the same sample as in (b) after thermal equilibration.

\subsection{Effect of Proton Saturation}

The two mechanisms proposed as an explanation of the "Birmingham" effects differ sharply with respect to the effect of manipulating the ${ }^{1} \mathrm{H}$ magnetization prior to dissolution. Quantum-rotor polarization effects are due to an imbalance between spin-isomer populations established by thermal equilibration at low temperature, and should be unaffected by manipulations of the ${ }^{1} \mathrm{H}$ magnetization in the cryogenic state, since this does not affect spin isomer populations. The nuclear Overhauser mechanism, on the other hand, relies on ${ }^{1} \mathrm{H}$ magnetization and is expected to be strongly affected by perturbations of the ${ }^{1} \mathrm{H}$ spins prior to dissolution.

The procedure shown in Figure 1(b) was used to test the effect of ${ }^{1} \mathrm{H}$ spin manipulations in the cryogenic state. A series of $51290^{\circ}$ pulses on the ${ }^{1} \mathrm{H}$ channel, each separated by $100 \mathrm{~ms}$, was applied to saturate the ${ }^{1} \mathrm{H}$ magnetization immediately before dissolution. This ${ }^{1} \mathrm{H}$ saturation burst is very short relative to the relaxation time constants in the cryogenic state, and is unlikely to influence the ${ }^{13} \mathrm{C}$ magnetization. The ${ }^{13} \mathrm{C}$ spectrum of unlabelled acetone, obtained after dissolution, transport, injection into the 11.7 $\mathrm{T}$ magnet, and application of a $90^{\circ}{ }^{13} \mathrm{C}$ pulse, is shown in Figure 2(c). The ${ }^{13} \mathrm{C}$ peak of the $\mathrm{CH}_{3}$ site displays a positive enhancement in this case.

Figure $3(\mathrm{~b})$ shows the ${ }^{13} \mathrm{C}$ spectrum of a $1: 1$ mixture of unlabelled acetone and $\left[1,2-{ }^{13} \mathrm{C}_{2}\right]$-acetone, subjected to the same procedure, but without applying ${ }^{1} \mathrm{H}$ decoupling during signal acquisition. This displays a multiplet with the same sign as the thermal equilibrium spectrum, and again without noticeable intensity distortions of the multiplet signal components.

We have obtained qualitatively similar results (i.e. no ${ }^{13} \mathrm{C}$ peak inversion) for the dissolution of acetone which had been equilibrated at cryogenic temperature outside the polarizer magnet.
These results strongly favour the nuclear Overhauser mechanism over the quantum-rotor polarization mechanism, as an explanation of the "Birmingham" observations.

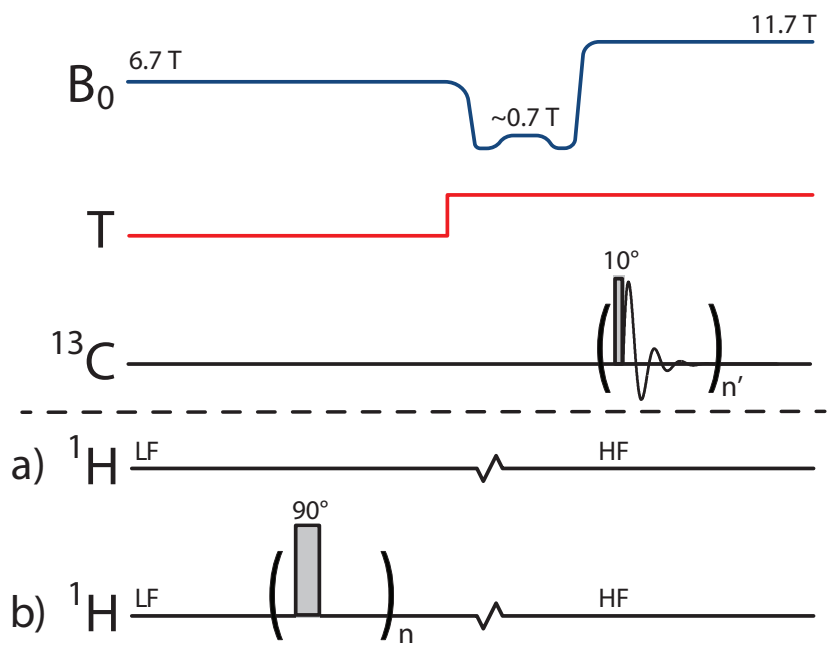

Figure 4: Experimental procedure for tracking the trajectory of the ${ }^{13} \mathrm{C}$ magnetization of the $\mathrm{CH}_{3}$ groups in acetone. After dissolution and transport to high field, and stabilisation of the solution, a series of $10^{\circ}$ ${ }^{13} \mathrm{C}$ pulses, each followed by two seconds of data acquisition, is applied. Fourier transformation of each free-induction decay leads to a separate ${ }^{13} \mathrm{C}$ spectrum. Other details are as in Figure 1.

\subsection{Magnetization Trajectories}

The procedures sketched in Figure 4 were used to track the trajectory of ${ }^{13} \mathrm{C}$ magnetization in the high-field magnet after dissolution and transport. A succession of ${ }^{13} \mathrm{C}$ free-induction decays was recorded by applying $10^{\circ}{ }^{13} \mathrm{C}$ pulses every two seconds. The integrals of the corresponding spectra over the $\mathrm{CH}_{3}$ spectral region were used to estimate the ${ }^{13} \mathrm{C}$ magnetization as a function of time. ${ }^{1} \mathrm{H}$ decoupling was not used, in order to avoid perturbations of the ${ }^{1} \mathrm{H}$ spins. The experiments were performed on a 1:1 mixture of unlabelled acetone and $\left[1,2-{ }^{13} \mathrm{C}_{2}\right]$-acetone, both without (a) and with (b) saturation of the ${ }^{1} \mathrm{H}$ magnetization before dissolution.

The resulting ${ }^{13} \mathrm{C}$ magnetization trajectories are shown in Figure 5, in which the enhancements were estimated by comparison with the thermal equilibrium spectra, as described in Section 2.3. The trajectories are very different when ${ }^{1} \mathrm{H}$ saturation pulses are not applied (a), or applied (b), prior to dissolution.

The trajectories are matched well by simulations of the nuclear spin evolution, performed using the SpinDynamica software package [19] (solid lines in Figure 5). These simulations assume isotropic rotational diffusion of the acetone molecules with a correlation time $\tau_{C}=3.2 \mathrm{ps}$, and internal methyl rotation with a correlation time $\tau_{R}=200 \mathrm{ps}$ (the simulations are insensitive to the value of $\tau_{R}$ in this regime). The correlation time $\tau_{C}$ is short enough to permit an extreme-narrowing approximation for the relaxation superoperator [20]. All dipole-dipole and chemical 
shift anisotropy terms, and all cross-correlations, are taken into account, although the effect of cross-correlations was found to be minor in this case. Similar calculations have been used to treat methyl long-lived states and quantumrotor-induced polarization in $\gamma$-picoline $[5,6]$, and longlived state relaxation in $\mathrm{CH}_{2} \mathrm{D}$ groups [21].

The simulations employ Liouville-space calculations of the nuclear spin dynamics, including the cross-relaxation processes, at a temperature of $300 \mathrm{~K}$ in a field of $11.7 \mathrm{~T}$. The simulations were fitted to the experimental data by adjusting the initial ${ }^{1} \mathrm{H}$ and ${ }^{13} \mathrm{C}$ polarisations at the start of the evolution, as well as the time origin, in order to take into account the delay between dissolution and the start of NMR signal acquisition. The initial proton polarization was set to zero in the simulation of experiments employing proton saturation prior to dissolution.

Best match simulations were obtained by setting the initial polarization levels to $506 \mathrm{ppm}$ for ${ }^{1} \mathrm{H}$ and $49.6 \mathrm{ppm}$ for ${ }^{13} \mathrm{C}$, and a shift of the initial time origin by $7.9 \mathrm{~s}$. The initial ${ }^{1} \mathrm{H}$ polarization level of $506 \mathrm{ppm}$ is 12.7 times larger than for thermal equilibrium in the high-field magnet, but 3.2 times smaller than for thermal equilibration in the polarizer at $4.2 \mathrm{~K}$ and $6.7 \mathrm{~T}$. Similarly, the initial ${ }^{13} \mathrm{C}$ polarization level of $49.6 \mathrm{ppm}$ is 4.95 times larger than that found for thermal equilibrium in the high-field magnet, but 8.3 times smaller than for full thermal equilibration in the polarizer. These discrepancies may be attributed to incomplete thermal equilibration in the polarizer before dissolution (especially for ${ }^{13} \mathrm{C}$ ), and transport losses.

The simulations show that a full explanation of the observed effects must take into account the ${ }^{13} \mathrm{C}$ magnetization as well as the ${ }^{1} \mathrm{H}$ magnetization established in the polarizer. When the ${ }^{1} \mathrm{H}$ magnetization is saturated prior to dissolution, a positive ${ }^{13} \mathrm{C}$ signal enhancement is generated at high temperature, simply because any ${ }^{13} \mathrm{C}$ magnetization built up at low temperature in the polarizer magnet is substantially retained during the transport process. When the ${ }^{1} \mathrm{H}$ magnetization is retained, on the other hand, cross-relaxation from the larger ${ }^{1} \mathrm{H}$ magnetization leads to a stronger, and negative, signal enhancement. This is consistent with the well-known dominance of double-quantum cross-relaxation processes for the dipole-dipole mechanism at short correlation times $[14,20]$.

\section{Discussion}

In summary, we have established that at least in the case of acetone, there is no need to invoke exotic quantum rotor effects in order to explain the negative enhancement of ${ }^{13} \mathrm{C}$ signals from proton-bearing carbon sites in dissolution NMR experiments. The observed effects arise from rather conventional cross-relaxation processes. The nuclear magnetization established by thermal equilibration under cryogenic conditions is far from equilibrium at a higher sample temperature. The establishment of equilibrium in the new physical conditions proceeds in part through heteronuclear

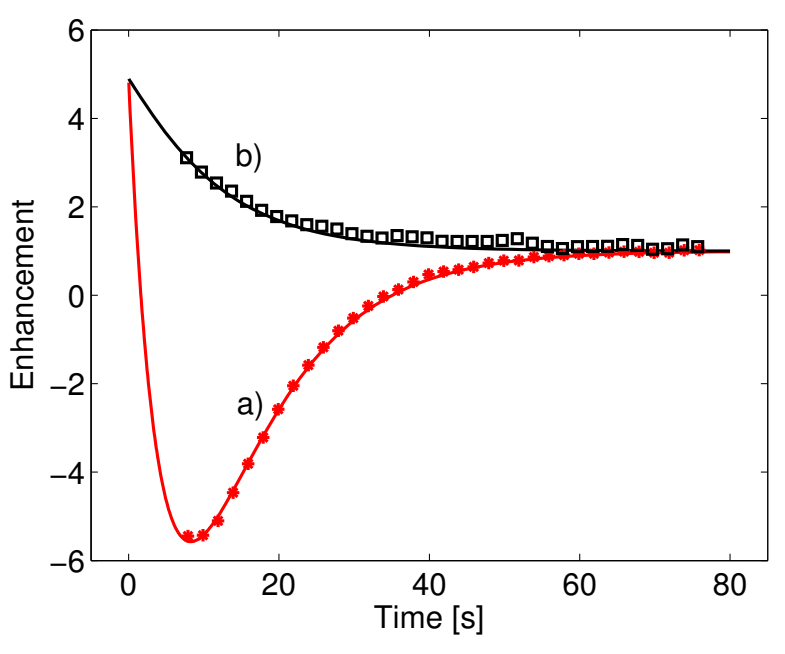

Figure 5: Experimental (symbols) and simulated (solid lines) trajectories of the ${ }^{13} \mathrm{C}$ magnetization for the $\mathrm{CH}_{3}$ groups of a 1:1 mixture of unlabelled acetone and $\left[1,2-{ }^{13} \mathrm{C}_{2}\right]$-acetone. (a, filled symbols) Data for the experiment in Figure 4(a). (b, open symbols) Data for the experiment in Figure 4(b). The solid lines are best-fit SpinDynamica simulations with a rotational correlation time of $\tau_{C}=3.2 \mathrm{ps}$ and initial Zeeman polarizations of $506 \mathrm{ppm}$ for ${ }^{1} \mathrm{H}$ and $49.6 \mathrm{ppm}$ for ${ }^{13} \mathrm{C}$ (see text). The initial ${ }^{1} \mathrm{H}$ polarization was set to zero for simulation (b).

cross-relaxation processes which lead to transient negative enhancements of ${ }^{13} \mathrm{C}$ magnetization. Effects of similar origin have been reported in dissolution-DNP experiments [22].

In this mechanism, the role of methyl groups is purely to act as a relaxation sink for ${ }^{1} \mathrm{H}$ spin-lattice relaxation under cryogenic conditions, allowing ${ }^{1} \mathrm{H}$ magnetization to build up at very low temperature.

The effects reported in this work are significantly smaller in magnitude than those reported by Ludwig et al. [1]. This may be partly due to differences in transport time and partly due to different polarizer conditions. The Birmingham study employed a lower polarizer temperature and also a lower polarizer field than we did, and the samples were typically allowed to rest in the polarizer for much longer than in our experiments. The low polarizer temperature of $1.4 \mathrm{~K}$ used in ref. [1], and the long waiting times in the polarizer, are both expected to lead to a stronger proton polarization than in our experiments, and hence a stronger Overhauser enhancement of the ${ }^{13} \mathrm{C}$ magnetization after dissolution. The use of a lower polarizer field works in the opposite direction, but it appears that the temperature effect dominates.

In some substances, Ludwig et al. [1] reported that the negative enhancement of ${ }^{13} \mathrm{C}$ signals after dissolution changed into a positive enhancement when the samples were left for very long times in the polarizer before dissolution. This mysterious effect might be explained by a strong differential in spin-lattice relaxation times for ${ }^{13} \mathrm{C}$ and ${ }^{1} \mathrm{H}$ under cryogenic conditions. At relatively short waiting times in the polarizer, ${ }^{1} \mathrm{H}$ magnetization is likely to dominate since ${ }^{1} \mathrm{H}$ spin-lattice relaxation is relatively fast. As discussed above, this gives rise to a negative en- 
hancement of ${ }^{13} \mathrm{C}$ magnetization after dissolution. At long waiting times in the polarizer, on the other hand, a substantial ${ }^{13} \mathrm{C}$ magnetization could build up, through relatively slow ${ }^{13} \mathrm{C} T_{1}$ processes. In this case, the direct ${ }^{13} \mathrm{C}$ polarization effect dominates after dissolution, and gives rise to positive ${ }^{13} \mathrm{C}$ signal enhancements.

\section{Acknowledgments}

The authors thank Ulrich Günther for generous and open-minded discussions, and Karel Kouřil and Giuseppe Pileio for insightful comments. This research was supported by the Engineering and Physical Sciences Research Council (EPSRC-UK), grant codes EP/N002482 and EP/L505067/1, the European Research Council (ERC), the Wolfson Foundation, and by Bruker (UK).

\section{References}

[1] C. Ludwig, M. Saunders, I. Marin-Montesinos and U. L. Günther, Proceedings of the National Academy of Sciences, 2010, 107, 10799-10803.

[2] M. Icker and S. Berger, J. Magn. Reson., 2012, 219, 1-3.

[3] M. Icker, P. Fricke and S. Berger, J. Magn. Reson., 2012, 223, 148-150.

[4] M. Icker, P. Fricke, T. Grell, J. Hollenbach, H. Auer and S. Berger, Magn. Reson. Chem., 2013, 51, 815-820.

[5] B. Meier, J.-N. Dumez, G. Stevanato, J. T. Hill-Cousins, S. S. Roy, P. Håkansson, S. Mamone, R. C. D. Brown, G. Pileio and M. H. Levitt, J. Am. Chem. Soc., 2013, 135, 18746-18749.

[6] J.-N. Dumez, P. Håkansson, S. Mamone, B. Meier, G. Stevanato, J. T. Hill-Cousins, S. S. Roy, R. C. D. Brown, G. Pileio and M. H. Levitt, J. Chem. Phys., 2015, 142, 044506.

[7] S. S. Roy, J.-N. Dumez, G. Stevanato, B. Meier, J. T. HillCousins, R. C. Brown, G. Pileio and M. H. Levitt, J. Magn. Reson., 2015, 250, 25-28.

[8] J.-H. Ardenkjær-Larsen, B. Fridlund, A. Gram, G. Hansson, L. Hansson, M. H. Lerche, R. Servin, M. Thaning and K. Golman, Proc. Natl. Acad. Sci. U.S.A., 2003, 100, 10158-10163.

[9] J. Haupt, Phys. Lett. A, 1972, 38, 389-390.

[10] A. Horsewill, Prog. Nucl. Magn. Reson. Spectrosc., 1999, 35, 359-389.

[11] M. Tomasselli, U. Meier, and B. H. Meier, J. Chem. Phys., 2004, 120, 4051-4054.

[12] R. M. Green, and A. J. Horsewill, Mol. Phys., 1986, 57, 887899.

[13] M. Prager and A. Heidemann, Chem. Rev., 1997, 97, 29332966.

[14] D. Neuhaus and M. Williamson, The Nuclear Overhauser Effect in Structural and Conformational Analysis, VCH, New York, 1989.

[15] J. Milani, B. Vuichoud, A. Bornet, P. Miéville, R. Mottier, S. Jannin and G. Bodenhausen, Review of Scientific Instruments, 2015, 86, 024101.

[16] A. Bornet, R. Melzi, A. J. Perez Linde, P. Hautle, B. van den Brandt, S. Jannin and G. Bodenhausen, The Journal of Physical Chemistry Letters, 2013, 4, 111-114.

[17] T. Cheng, M. Mishkovsky, J. A. M. Bastiaansen, O. Ouari, P. Hautle, P. Tordo, B. V. D. Brandt and A. Comment, NMR Biomed., 2013, 26, 1582-1588.

[18] A. Shaka, J. Keeler, T. Frenkiel and R. Freeman, Journal of Magnetic Resonance (1969), 1983, 52, 335-338.

[19] C. Bengs and M. H. Levitt, Magn. Reson. Chem., 2017.

[20] A. Abragam, Principles of Nuclear Magnetism, Clarendon Press, Oxford, 1961.
[21] S. J. Elliott, L. J. Brown, J. N. Dumez, and M. H. Levitt, J. Magn. Reson., 2016, 272, 87-90.

[22] K. J. Donovan, A. Lupulescu and L. Frydman, ChemPhysChem, 2014, 15, 436-443. 